\title{
Electrochemical Evaluation of the Corrosion Grade in Reinforced Steel in the Presence of Admixtures
}

\author{
R. Velázquez-González ${ }^{*}$, M.E. Acosta-Lomelí, C. Gaona-Tiburcio, \\ F. Almeraya-Calderón, A. Martínez-Villafañe \\ Centro de Investigación en Materiales Avanzados, S.C. \\ División de Deterioro de Materiales e Integridad Estructural / Grupo de Corrosión \\ Miguel de Cervantes 120, Complejo Ind. Chihuahua \\ Chihuahua, Chih. México
}

Received 27 May 2004; accepted in revised form 2 September 2004

\begin{abstract}
In the present paper we have carried out a study in order to determine the grade of corrosion affectation in concrete structures reinforced by the incorporation of additives, using electrochemical techniques and analyzing the respective effect on their mechanical properties. Quantitative and qualitative determination of their properties has been done (chemical analysis of the additives and steel, resistance to the compression, and flexural strength, porosity, and water permeability). The methodology was carried out according to ASTM standards and the action of the admixtures was evaluated using corrosion potentials and corrosion rates. The carbonatation and penetration of chlorides were also determined. The study was supplemented with a morphologic characterization by means of a scanning electron microscope. Specimens with and without reinforcement were elaborated, three of each being tested in order to obtain an average. Prismatic specimens without reinforcement were also casted with the following chemical additives: air-entraining, plasticizers, retardants, accelerating, coloring and fly ash. After curing, they were exposed to an aggressive atmosphere with sodium chloride. The general performance of the specimens after the period of exposition is good, since practically all of them developed very low corrosion rates, between $0.55 \times 10^{-4}$ and 4.76 $\times 10^{-4} \mu \mathrm{A} / \mathrm{cm}^{2}$.
\end{abstract}

Keywords: corrosion, admixture, reinforcement, electrochemical, chlorides.

\footnotetext{
* Corresponding author. E-mail address: ricardo.velazquez@cimav.edu.mx
} 


\section{Introducción}

Una parte muy importante de las estructuras de concreto se encuentra reforzada con acero y la corrosión de estos refuerzos es la causa principal del deterioro de las mismas. El deterioro causado por la corrosión no tiene descanso, la ciencia y la tecnología continúan realizando nuevos estudios y metodologías para desarrollar materiales más resistentes [1].

El concreto hidráulico es un producto resistente a compresión y frágil a flexión y además tiende a agrietarse desde su colocación, haciendo esto inevitable el uso de materiales que nos ayuden a mejorar las propiedades mecánicas del concreto hidráulico. Es por esto que la utilización de acero nos ayuda a aumentar la capacidad de carga del concreto a flexión.

Los aditivos han proporcionado al concreto características que favorecen el uso de este en los diferentes climas. Es por eso que el presente trabajo realiza un estudio comparativo para determinar el daño en las estructuras de concreto armado por la influencia de los aditivos como iniciadores de la corrosión así como en sus propiedades mecánicas. Los aditivos empleados son aireante, plastificante, retardante, acelerante, colorante y fly ash.

En muchos países se ha prohibido el uso de $\mathrm{CaCl}_{2}$ como acelerante del fraguado por su elevada agresividad en la corrosión del acero de refuerzo [2]. En las regiones tropicales en México, la principal fuente de contaminación de cloruros es la sal proveniente de la brisa marina en las costas [3], que puede desencadenar la corrosión localizada en el acero de refuerzo del concreto. Los cloruros también pueden ingresar al concreto a través de aditivos acelerantes de fraguado, 0 reductores de agua, los cuales suelen contener cloruros en su composición.

El concreto confiere al acero una protección de doble naturaleza, siendo en primer lugar una barrera física que lo aísla del medio ambiente, y en segundo lugar el líquido encerrado en los poros del concreto es un electrolito que lo pasiva de forma duradera. El grado de protección que puede dar un concreto es, con frecuencia una función de calidad, del espesor del recubrimiento y de una metodología adecuada de construcción. Sin embargo apesar de de la protección contra la corrosión que proporciona el concreto, se reportan casos de corrosión 
del acero ahogado en el concreto. La corrosión en ambientes marinos, urbanos, rurales o industriales es un problema presente.

La corrosión del acero de refuerzo en las estructuras de concreto que ocurre por la destrucción de la capa pasivante formada naturalmente sobre el acero embebido en concreto, tienen dos causas principales: 1.- la presencia de una cantidad suficiente de cloruros (añadidos durante la fabricación del concreto o por la penetración del exterior) u otros iones despasivantes en contacto con el refuerzo; 2.- la disminución de la alcalinidad del concreto cuando éste reacciona con sustancias ácidas del medio ambiente.

Los iones despasivantes provocan una corrosión de tipo localizado, mientras que la reducción de la alcalinidad del concreto permite la disolución completa de la capa pasivante y, por lo tanto, ocasiona una corrosión de tipo generalizado [4].

\section{Desarrollo experimental}

La elaboración de especimenes fue de 63 de los cuales 18 fueron con aditivos y con acero, 18 sin acero con aditivo, 3 sin acero sin aditivo, y 3 con acero sin aditivo; estos 42 especimenes corresponden a cilindros de $30 \times 15 \mathrm{~cm}$ de diámetro. Además se hicieron 18 vigas de $15 \times 15 \times 50 \mathrm{~cm}$ con aditivo sin acero, y 3 vigas sin aditivo y sin acero. Todos los especimenes se realizaron de acuerdo a la Norma (ASTM C-192) [5]. Los aditivos empleados en los especimenes fueron aireante, plastificante, retardante, acelerante, colorante y fly ash. El concreto trabajado tuvo una resistencia de $\mathrm{f}^{\prime} \mathrm{c}=250 \mathrm{~kg} / \mathrm{cm}^{2}$ con una relación agua/cemento de 0.58 , el tamaño máximo del agregado de $3 / 4$ " , con acero de refuerzo de $3 / 8$ ".

Se tomó lectura del $\mathrm{pH}$ de la mezcla de concreto fresco en los diferentes especimenes y se tomó la temperatura de cada uno de los especimenes [6], se realizó análisis químico cualitativo y cuantitativo por medio de ICP y analizador elemental CHNS-O de cada uno de los aditivos, así como del cemento y del acero. Se realizó un análisis por medio de microscopía electrónica de barrido (JEOL JMS-5800LV / EDAX) de la forma de la partícula del colorante, del fly ash y del cemento. Se realizaron pruebas de resistencia mecánica a la flexión 
(ASTM C78) [7] y a la compresión (ASTM C39) [8]. Se determinó el contenido de iones cloruro solubles contenidos en agua como lo indica la Norma (ASTM D-1411) [9] al terminar la etapa de curado. Se determino la profundidad de carbonatación en el concreto por el método de vía húmeda con indicador ácidobase después del curado (ACI 201) [10]. Se determinó la porosidad y el porcentaje de agua absorbida de muestras de concreto con los diferentes aditivos, como lo indica la Norma (ASTM C-642-97)[11].

En el proceso de curado se realizó medición de potenciales a los 18 cilindros de concreto con acero y con aditivo, y a 3 cilindros de concreto con acero y sin aditivo con electrodo de referencia de $\mathrm{Cu} / \mathrm{CuSO}_{4}$ (CSE) y un voltímetro digital, utilizando las mismas varillas de refuerzo como electrodo de trabajo.

A $\operatorname{los} 21$ cilindros de concreto se le realizaron pruebas electroquímicas con el equipo de corrosión Auto Tafel VI.73 de ACM Instruments utilizando la técnica de curvas de polarización con los especimenes inmersos en salmuera al $3.5 \%$, por un período de 8 meses [12].

Terminada la fase experimental se realizó un análisis al acero de refuerzo de los diferentes especimenes por medio de microscopía electrónica de barrido (JEOL JMS-5800LV / EDAX).

Tabla 1. Análisis químico del acero.

\begin{tabular}{|c|c|}
\hline \multicolumn{2}{|c|}{ Analizador elemental CHNS-O } \\
\hline Elemento & Acero al carbono \\
\hline $\mathrm{C} \%$ & 0.1688 \\
\hline $\mathrm{Mn} \%$ & 1.0758 \\
\hline $\mathrm{S} \%$ & 0.0211 \\
\hline $\mathrm{Si} \%$ & 0.1452 \\
\hline
\end{tabular}

\section{Resultados y discusión}

Los datos obtenidos del $\mathrm{pH}$ del concreto fresco oscilan en un intervalo de 12 a 13 , la temperatura de la mezcla fue de $23^{\circ} \mathrm{C}$ a $29^{\circ} \mathrm{C}$.

Los resultados del análisis químico cualitativo y cuantitativo por medio del analizador elemental CHNS-O, se muestran en la Tabla 1 para el acero. 
En la Tabla 2 se presenta el resultado del análisis químico cualitativo por ICP y cuantitativo por absorción atómica para los aditivos en la cual se muestran los elementos que lo componen cumpliendo con esto la normativa.

Tabla 2. Reporte de análisis químico*.

\begin{tabular}{|c|c|c|c|c|c|c|}
\hline Elemento & Colorante & Fly ash & Plastificante & Retardante & Acelerante & Aireante \\
\hline $\mathrm{Al} \%$ & 0.2188 & 3.688 & - & - & - & - \\
\hline $\mathrm{Ba} \%$ & 0.0051 & 0.0624 & - & 0.0003 & 0.0003 & - \\
\hline $\mathrm{Ca} \%$ & 4.610 & 2.876 & - & 0.3372 & 11.99 & 0.0093 \\
\hline $\mathrm{Cr} \%$ & 0.0383 & 0.0035 & & - & - & - \\
\hline $\mathrm{Cu} \%$ & 0.0015 & 0.0047 & 0.0043 & 0.0001 & - & 0.0001 \\
\hline $\mathrm{Fe} \%$ & 21.8036 & 1.3150 & 0.0017 & 0.0035 & 0.0006 & 0.0015 \\
\hline $\mathrm{K} \%$ & 0.0772 & 0.2845 & 0.0081 & 0.1550 & 0.2175 & 0.0077 \\
\hline $\mathrm{Mg} \%$ & 0.0887 & 0.5143 & 0.0202 & 0.0308 & 0.0024 & 0.0042 \\
\hline $\mathrm{Mn} \%$ & 0.0308 & 0.0374 & - & 0.0020 & - & 0.0003 \\
\hline $\mathrm{Na} \%$ & 0.0603 & 0.0770 & 0.5948 & 0.0904 & 0.2609 & 1.0605 \\
\hline $\mathrm{Si} \%$ & 0.7622 & 10.4266 & - & 0.0001 & - & 0.0046 \\
\hline $\mathrm{Sr} \%$ & 0.0058 & 0.0455 & - & 0.0005 & 0.0070 & - \\
\hline $\mathrm{Ti} \%$ & 0.0187 & 0.4174 & - & - & - & - \\
\hline $\mathrm{V} \%$ & 0.0075 & 0.0080 & - & - & - & - \\
\hline $\mathrm{Zn} \%$ & 0.0295 & 0.0011 & - & 0.0001 & - & 0.0010 \\
\hline
\end{tabular}

* El valor del análisis $100 \%$ atómico.

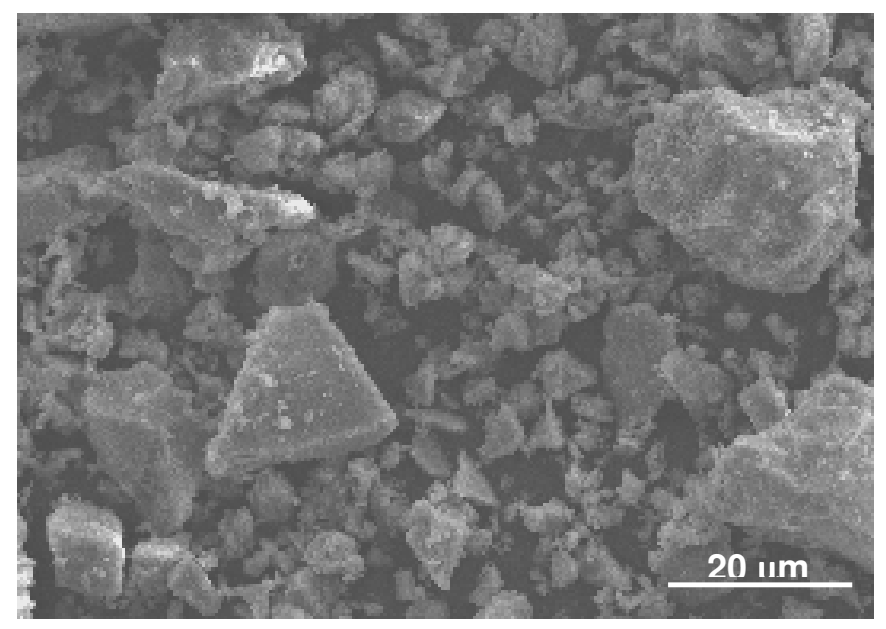

Figura 1. Cemento.

La morfología de los aditivos colorante, fly ash así como del cemento, se puede apreciar en las fotomicrografías siguientes. 
En el análisis de la forma de la partícula del cemento se aprecian partículas con amplia variación dimensional y de superficies con gran cantidad de aristas, ver Fig. 1.

En cambio la partícula del colorante presenta una forma alargada y de dimensiones uniformes, ver Fig. 2.

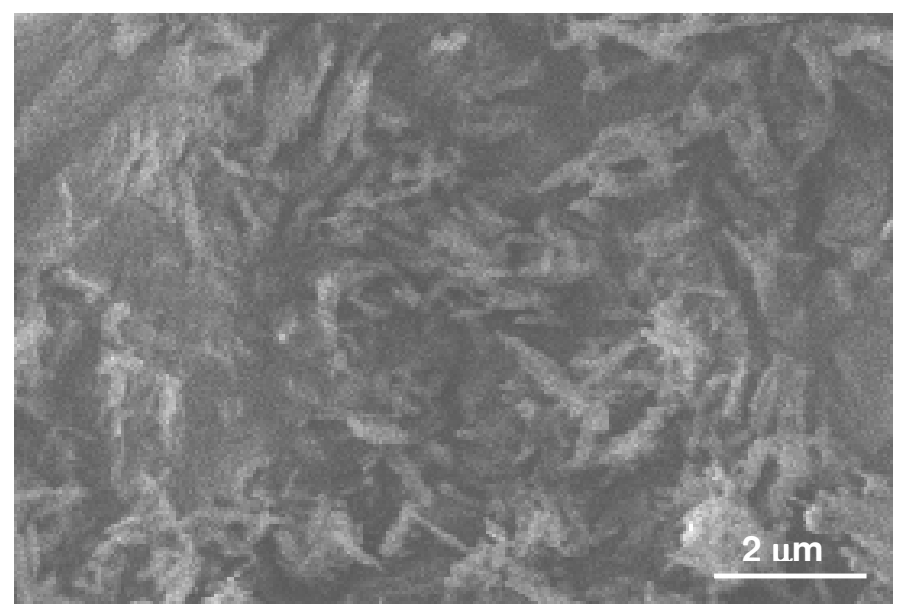

Figura 2. Colorante.

En la partícula del fly ash tipo F, se observa la forma esférica que lo caracteriza, ver Fig. 3.

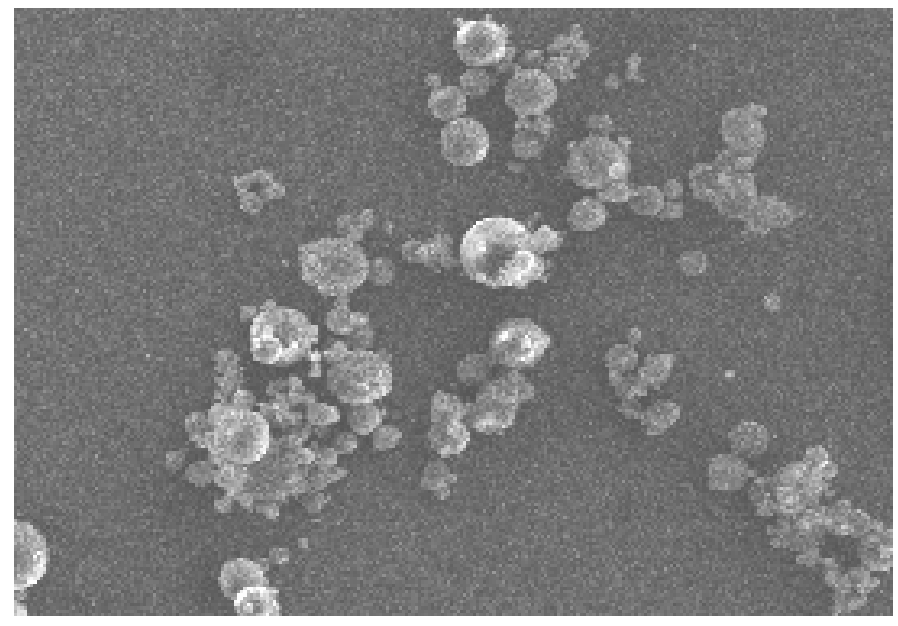

Figura 3. Fly ash tipo F.

El contenido de iones cloruro solubles contenidos en agua finalizada la etapa de curado se reporta en la Tabla 3, para cada uno de los aditivos. De acuerdo al 
criterio de riesgo para la iniciación de corrosión con relación a los rangos de CCRIT [13] los valores $<0.4 \%$ se consideran insignificantes.

Tabla 3. Análisis de iones cloruro.

\begin{tabular}{|c|c|}
\hline Muestra & \% Cl \\
\hline Concreto & 0.04314 \\
\hline Colorante & 0.0431 \\
\hline Fly ash & 0.0162 \\
\hline Plastificante & 0.0646 \\
\hline Retardante & 0.0539 \\
\hline Acelerante & 0.0539 \\
\hline Aireante & 0.0270 \\
\hline
\end{tabular}

En la Tabla 4 se muestran los resultados del \% de absorción de agua de las muestras de concreto con los diferentes aditivos de acuerdo a la Norma ASTM C 642-97, en la que se tiene una correlación con el porcentaje de porosidad del concreto; de acuerdo a los valores obtenidos y el tiempo de saturación podemos concluir que los concretos ensayados tienen una baja absorción que es lo que se esperaba [14]. En esta misma Tabla se presenta el porcentaje de porosidad de las muestras de concreto con los diferentes aditivos; de acuerdo al criterio de evaluación [15] del 10 al $15 \%$ indica un concreto de moderada calidad.

Tabla 4. Resultado de porosidad y absorción de agua.

\begin{tabular}{|c|c|c|}
\hline Muestra & \% Absorcion & \% Porosidad \\
\hline Concreto & 5.50 & 12.54 \\
\hline Colorante & 5.85 & 13.40 \\
\hline Fly ash & 5.40 & 12.34 \\
\hline Plastificante & 5.53 & 12.95 \\
\hline Retardante & 5.57 & 12.91 \\
\hline Acelerante & 6.10 & 14.05 \\
\hline Aireante & 6.28 & 13.69 \\
\hline
\end{tabular}


Durante el proceso de curado se realizó medición de potenciales a los 18 cilindros de concreto con acero y con aditivo, y a 3 cilindros de concreto con acero y sin aditivo, con electrodo de referencia de $\mathrm{Cu} / \mathrm{CuSO}_{4}$ (CSE), y un voltímetro digital, utilizando las mismas varillas de refuerzo como electrodo de trabajo. Los valores de potencial en este caso variaron de $-100 \mathrm{mV} \mathrm{a}-650 \mathrm{mV}$, lo que indica que existe la posibilidad, de que el material se este corroyendo.

En la etapa después del curado la medición de potenciales de dichos especimenes es de $-150 \mathrm{mV}$ a $-900 \mathrm{mV}$, indicando la probabilidad de que el material se esta corroyendo.

$\mathrm{Si}$ los potenciales medidos, con respecto al electrodo de referencia $\mathrm{Cu} / \mathrm{CuSO}_{4}$ (CSE) son más positivos que $-200 \mathrm{mV}$, el riesgo de que ocurra corrosión es del $10 \%$, si están entre $-200 \mathrm{mV}$ y $-350 \mathrm{mV}$, se tiene una incertidumbre y si son más negativos que $-350 \mathrm{mV}$, se tiene una probabilidad del $90 \%$ de que se esté corroyendo el acero de acuerdo a la Norma ASTM C-876-91[16].

Posterior al curado se realizaron pruebas electroquímicas en los 21 cilindros de concreto, empleando el equipo de corrosión Auto Tafel VI.73 de ACM Instruments. Se utilizó la técnica de curvas de polarización. Estos especimenes se mantienen inmersos en salmuera al $3.5 \%$ (por un período de 8 meses).

En las Fig. 4 a 6 se presentan las curvas de polarización obtenidas a distintos tiempos de exposición, para el concreto natural y los concretos elaborados con los distintos aditivos. En la etapa de curado, Fig. 4, se aprecia que los especimenes con fly ash, plastificante, retardante, acelerante y aireante tienen el mismo comportamiento y velocidad de corrosión, a excepción de los concretos natural y con colorante, con intensidades de corrosión menores y cuyos potenciales se encuentran entre $-400 \mathrm{mV} \mathrm{y}-600 \mathrm{mV}$.

Al exponer los especimenes al medio salino, Fig. 5, se aprecia como las intensidades de corrosión de los concretos con aditivo empiezan inmediatamente a ser mayores que la del concreto natural. Pero tras cuatro meses de exposición, se observan dos comportamientos distintos, uno agrupando colorante, fly ash, plastificante, retardante, acelerante, y aireante, en tanto el concreto natural mantiene su tendencia separado del grupo. 


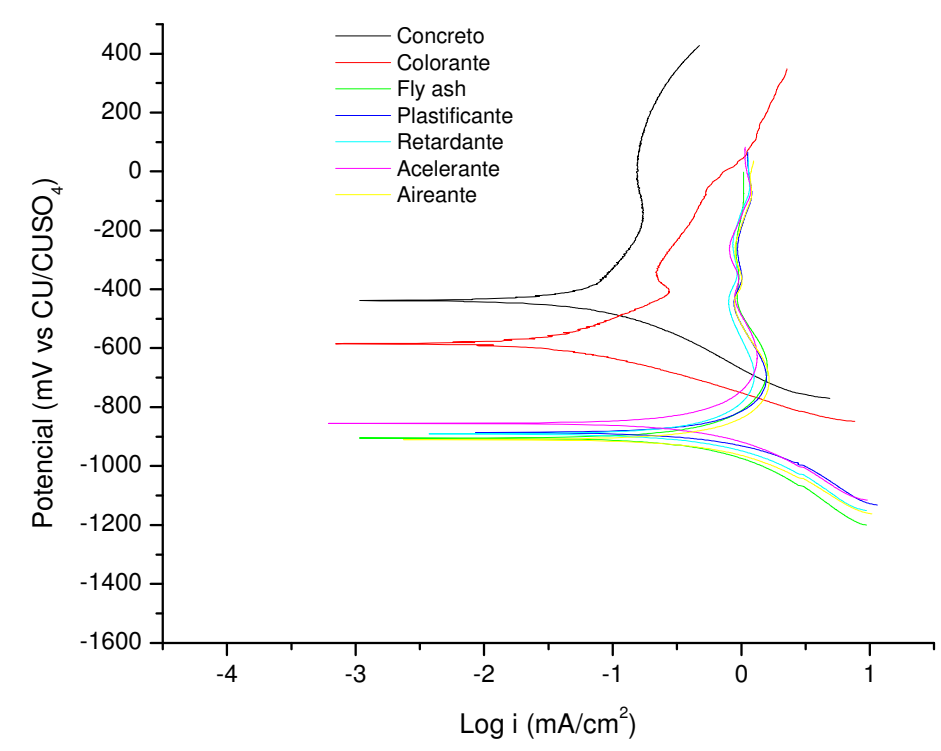

Figura 4. Curvas de polarización obtenidas durante el periodo de curado.

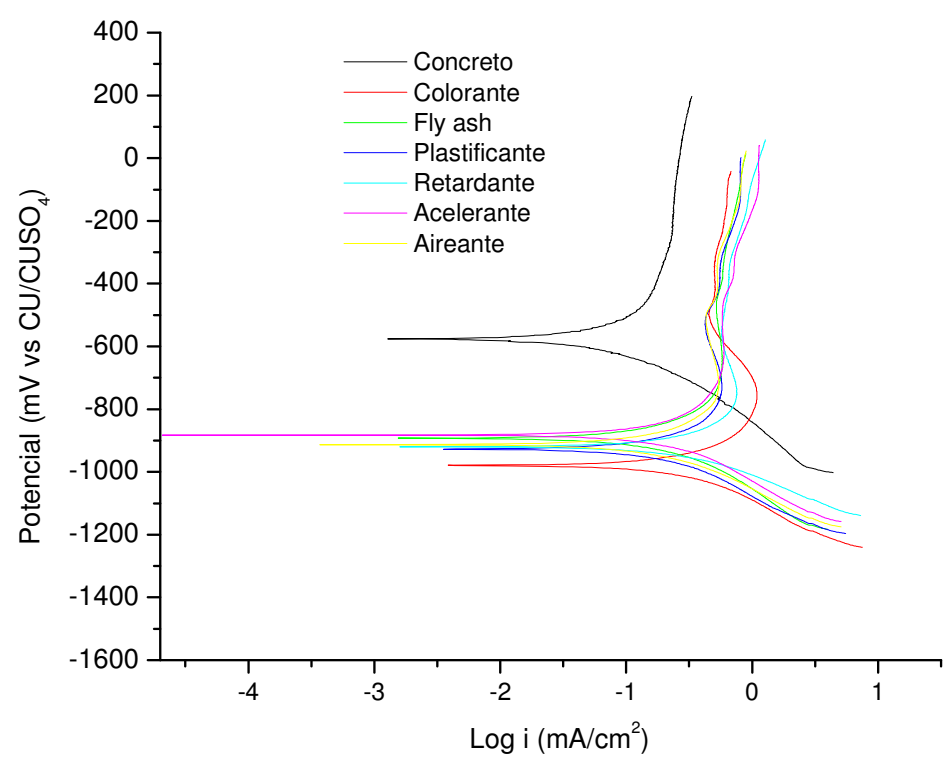

Figura 5. Curvas de polarización obtenidas tras 4 meses de exposición en salmuera. 
Tras 8 meses de exposición en la Fig. 6, se aprecia como las intensidades de corrosión de los concretos natural y fly ash son menores. Siendo mayores en los especimenes con colorante, plastificante, retardante, acelerante, y aireante.

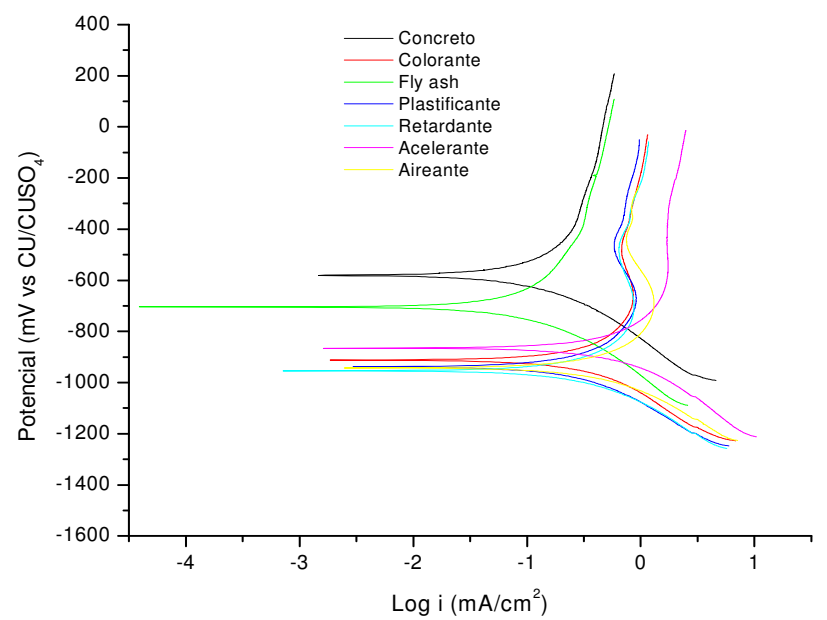

Figura 6. Curvas de polarización obtenidas tras ocho meses de exposición al medio salino.

En la Tabla 5 se muestran las velocidades de corrosión que corresponden a los gráficos de curvas de polarización mostrados con anterioridad. Se observa que los concretos con aditivos tienen una mayor velocidad de corrosión, a excepción del concreto con fly ash y concreto natural. El proceso de pasivación es claramente visualizado para los concretos con plastificante, retardante, aireante y colorante.

Las velocidades de corrosión corresponden a un nivel de corrosión bajo $(<0.1$ $\mu \mathrm{A} / \mathrm{cm}^{2}$ despreciable) [15].

Tabla 5. Resultado de velocidades de corrosión.

\begin{tabular}{|l|c|c|c|}
\hline \multirow{2}{*}{ Espécimen } & \multicolumn{3}{c|}{ Velocidad de corrosión $/\left(\boldsymbol{\mu A} / \mathbf{c m}^{2}\right)$} \\
\cline { 2 - 4 } & Curado & $\mathbf{4}$ meses & $\mathbf{8 ~ m e s e s}$ \\
\hline Concreto & $1.11 \times 10^{-4}$ & $0.74 \times 10^{-4}$ & $7.95 \times 10^{-4}$ \\
\hline Colorante & $3.10 \times 10^{-4}$ & $1.66 \times 10^{-4}$ & $1.32 \times 10^{-4}$ \\
\hline Fly ash & $1.54 \times 10^{-4}$ & $0.80 \times 10^{-4}$ & $0.55 \times 10^{-4}$ \\
\hline Plastificante & $4.76 \times 10^{-4}$ & $1.04 \times 10^{-4}$ & $1.27 \times 10^{-4}$ \\
\hline Retardante & $3.25 \times 10^{-4}$ & $1.41 \times 10^{-4}$ & $1.25 \times 10^{-4}$ \\
\hline Acelerante & $1.74 \times 10^{-4}$ & $0.96 \times 10^{-4}$ & $2.39 \times 10^{-4}$ \\
\hline Aireante & $0.42 \times 10^{-4}$ & $0.93 \times 10^{-4}$ & $2.10 \times 10^{-4}$ \\
\hline
\end{tabular}


Análisis de la morfología del acero de los especimenes de concreto utilizados en la experimentación, fotomicrografía de zonas con aparente corrosión, como se muestra en las siguientes figuras.

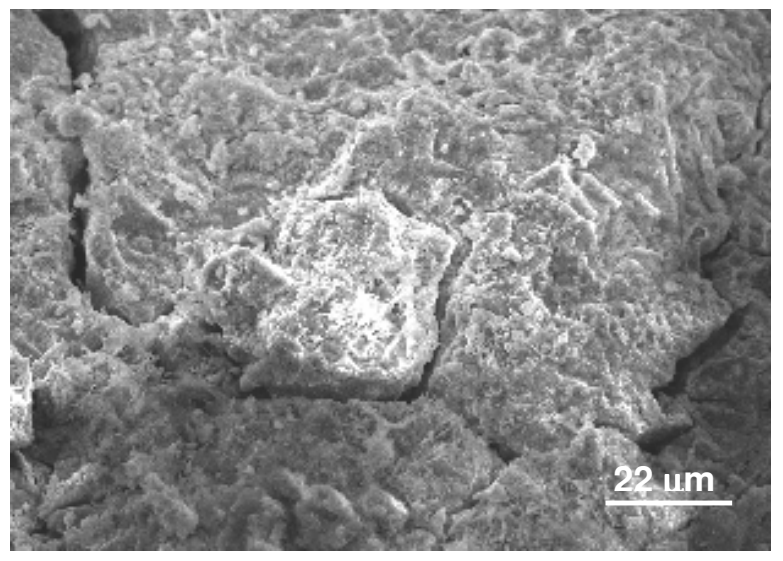

Figura 7. Concreto sin aditivo con una aproximación de 1000x.

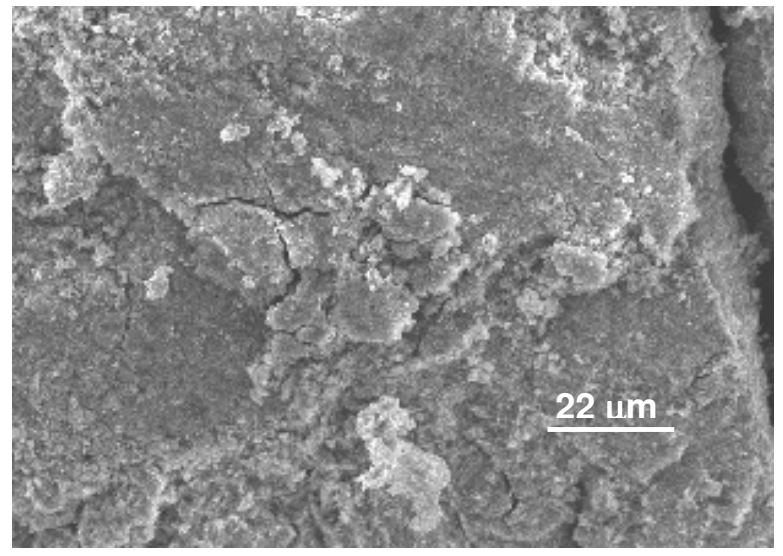

Figura 8. Concreto con aditivo colorante con una aproximación de 1000x.

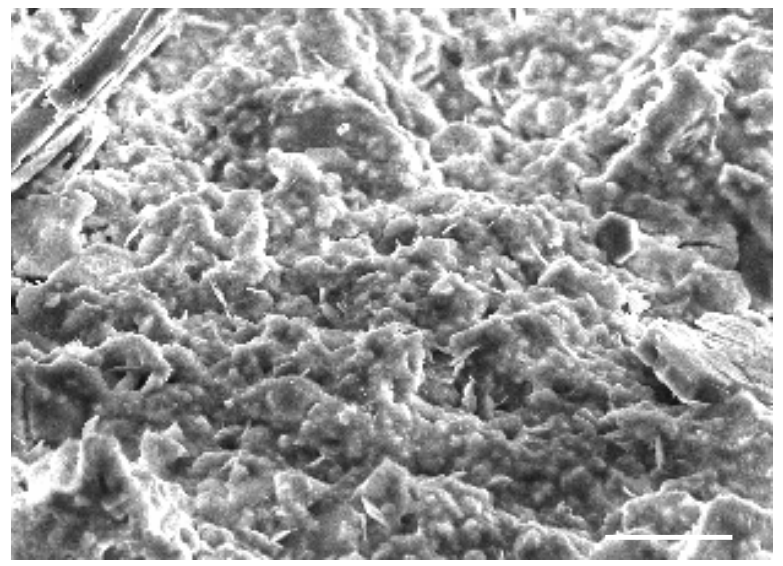

Figura 9. Concreto con aditivo fly ash con aproximación de 1000x. 


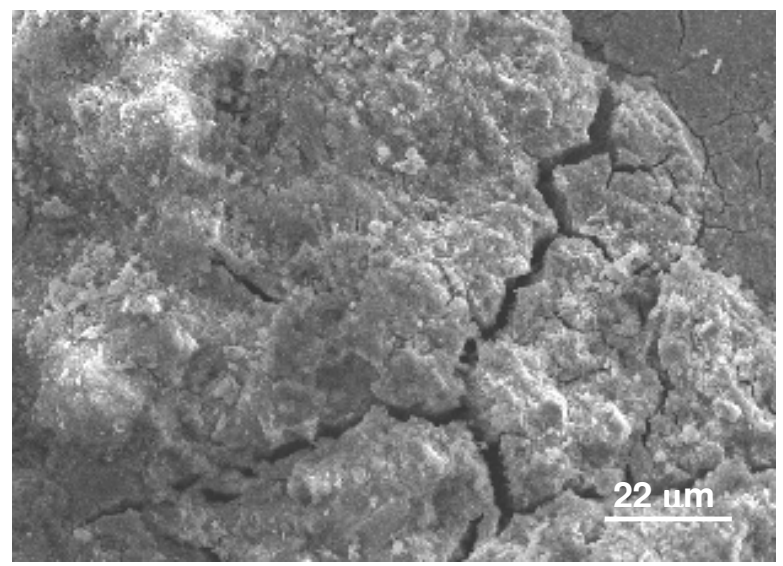

Figura 10. Concreto con aditivo plastificante con aproximación de 1000x.

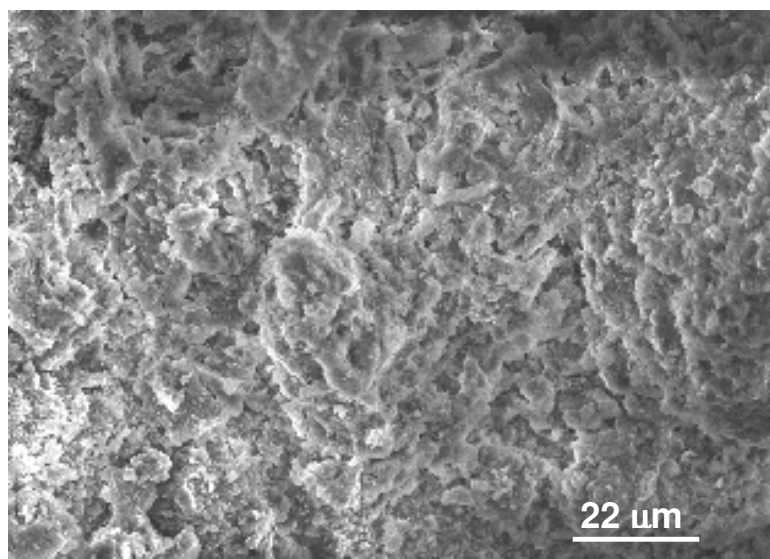

Figura 11. Concreto con aditivo retardante con aproximación de 1000x.

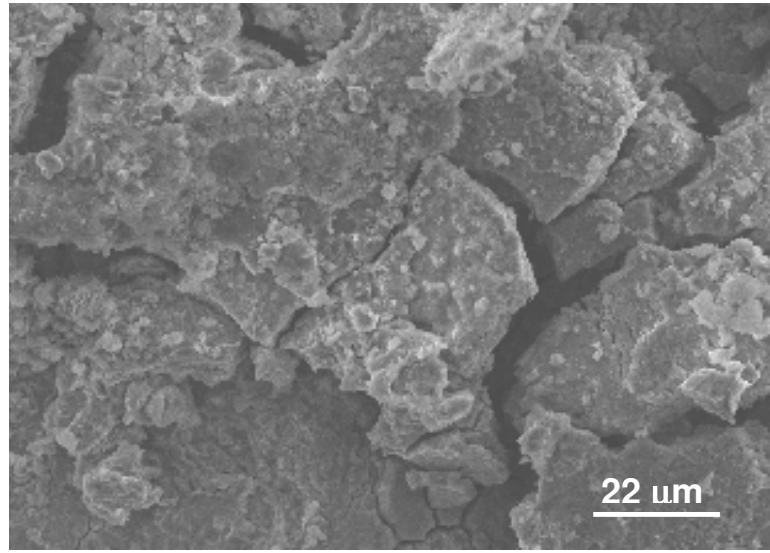

Figura 12. Concreto con aditivo acelerante con aproximación de 1000x. 


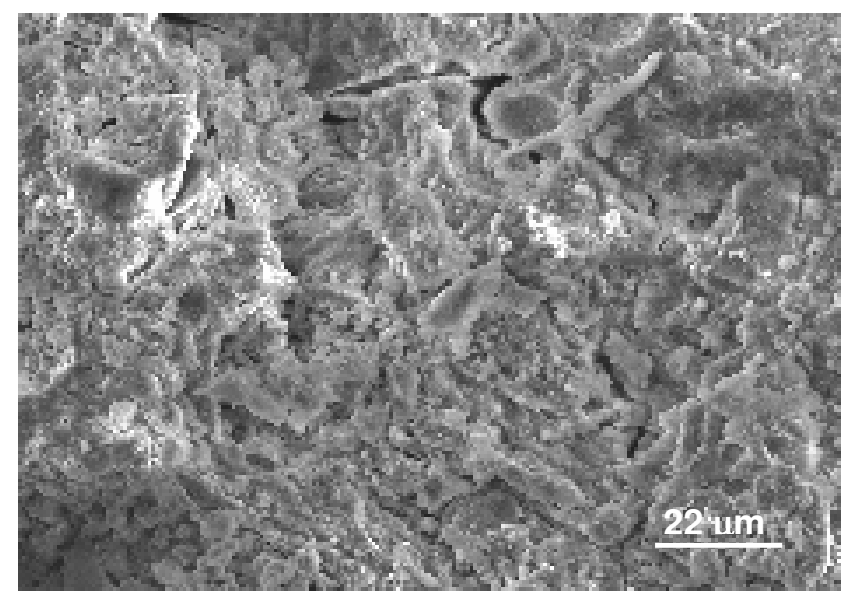

Figura 13. Concreto con aditivo aireante con aproximación de 1000x.

\section{Conclusiones}

Se realizó una completa caracterización de las propiedades físicas, químicas y mecánicas de los especimenes elaborados, encontrando que el colorante y aireante son los que mas afectan la resistencia mecánica del concreto. Y por otro lado, se observó que los especimenes menos afectados por la absorción de agua y la porosidad fueron el concreto natural y el fly ash.

Respecto a la evaluación de su desempeño en el medio salino, se pudo observar que el colorante, plastificante, retardante, acelerante, y aireante, se comportan de una manera similar, mostrando un mejor comportamiento el fly ash casi similar al concreto natural el cual ofrece una protección natural por sí solo al acero de refuerzo. El concreto con fly ash tipo f mejoró sus características por el acomodo de sus partículas en la mezcla. En términos generales la calidad de los agregados, la relación agua/cemento y el aditivo empleado juegan un papel muy importante en la elaboración de las mezclas y los resultados obtenidos. El desempeño general de los especimenes tras ocho meses de exposición es muy bueno, ya que prácticamente todos desarrollan velocidades de corrosión entre $0.55 \times 10^{-4}$ $\mu \mathrm{A} / \mathrm{cm}^{2}$ y $4.76 \times 10^{-4} \mu \mathrm{A} / \mathrm{cm}^{2}$ (velocidades muy bajas) que de acuerdo a la Red Durar, representan un nivel de corrosión bajo. 


\section{Agradecimientos}

Se agradece al Cimav por su apoyo, a Conacyt por la beca otorgada, a Coprechisa por la donación de los materiales.

\section{Evaluacion Electroquimica del Grado de Corrosion en Acero de Refuerzo en Presencia de Aditivos}

\section{Resumen}

En el presente trabajo se realizó un estudio para determinar el grado de afectación por corrosión en estructuras de concreto reforzado por la incorporación de aditivos, empleando técnicas electroquímicas y analizando el efecto de estos en sus propiedades mecánicas. Se hizo la determinación cuantitativa y cualitativa de sus propiedades (análisis químico de los aditivos y del acero, resistencia a la compresión, ensayos de flexión, porosidad y permeabilidad), de las cantidades óptimas para su funcionamiento adecuado de acuerdo a la normatividad ASTM, se evaluó el desempeño de los aditivos y se midieron los potenciales y las velocidades de corrosión. También se hicieron determinaciones de carbonatación y penetración de cloruros. El estudio se complementó con caracterización morfológica empleando microscopía electrónica de barrido. Se realizaron especimenes en series de tres cilindros para efectos de reproducibilidad: $\sin$ y con refuerzo, así como especimenes prismáticos sin refuerzo, todos con la incorporación de aditivos químicos: aireantes, plastificantes, retardantes y acelerantes de fraguado, colorantes y cenizas volantes (fly ash). Después del curado fueron expuestos a un ambiente agresivo con cloruro de sodio. El desempeño general de los especimenes tras el período de exposición es bueno, ya que prácticamente todos desarrollaron velocidades de corrosión entre $0.55 \times 10^{-4}$ y $4.76 \times 10^{-4} \mu \mathrm{A} / \mathrm{cm}^{2}$, siendo velocidades muy bajas.

Palabras clave: corrosión, aditivo, refuerzo, electroquímica, cloruros.

\section{Referencias}

1. Construcción y Tecnología, Instituto Mexicano del Cemento y del Concreto, A.C., Junio 2000.

2. C.R. Bonilla: "Recubrimientos anticorrosivos, su selección, aplicación e inspección", México (1986).

3. P.C. Borges, R.C. Salazar, J.J.P. Carpio, J.G. Llongueras, P.R.J. Helene, W.L. González, J.P.F. Enio, M.A.S. Barbudo, L.V. Muleshkova, “Corrosión en Estructuras de Concreto Armado" Instituto Mexicano del Cemento y del Concreto, A.C. Primera edición, 1998. 
4. NACE, "Corrosion Basics. An Introduction", National Association of Corrosion Engineers (NACE), U.S.A. (1984).

5. ASTM C 192/ C 192M- 98 "Standard Test Method for Production and Cured Specimens of Test Concretes in the Laboratory"

6. W. López, J.A. González, C. Andrade, "Influence of temperature on the service life of rebars". Cem. Conc. Res., Vol. 23, (1993): 99. 11.30-1140.

7. ASTM C78 "Standard Test Methods for Flexural Strength of Concrete" (Using simple Beam with Third-Point Loading). ASTM. U.S.A., 15 de abril de 1994, 3 págs.

8. ASTM C39 "Standard Test Methods for Strength of Cylindrical Concrete Specimens” ASTM U.S.A., 15 de noviembre de 1994, 5 págs.

9. ASTM D 1411 - 99 "Standard Test Methods for Water-Soluble Chlorides Present as Admixtures in Graded Aggregate Road Mixes"

10. ACI 201 Committee 201, "Guide to durable concrete". Report ACI 201R, American Concrete Institute, Detroit, EUA, 1982.

11. ASTM C 642-97 "Standard Test Method for Density, Absorption, and Voids in Hardened Concrete"

12. J.M. Bastidas, “Técnicas Electroquímicas de Estudio de la Corrosión”, en U.E.I. de Corrosión y Protección del C.S.I.C., Retrospectivas de los Estudios de Corrosión en España en el periodo de 1960-1990, España (1991).

13. A. Torres Acosta, "Diseño de estructuras de concreto con criterios de durabilidad" IMT, 2001, México.

14. P.A. Muhammed Basheer, "Handbook of Analytical Techniques in Concrete Science and Technology. Principles, Techniques and Applications", Edited by V.S. Ramachandran, J.J. Beaudoin, (C) 2001 William Andrew Publishing/Norwich, New York, U.S.A., 964 pgs.

15. O.T. Rincón, A.R. Carruyo, C. Andrade, P. Helene, I. Díaz, DURAR "Manual de inspección, evaluación y diagnóstico de corrosión en estructuras de hormigón armado", CYTED Red Temática XV.B Durabilidad de la Armadura (2000). 
16. ASTM C-876-91: "Standard Test Method for Half-Cell Potentials of Uncoated Reinforcing Steel in Concrete", American Society for Testing and Materials, Philadelphia, USA (1991). 